\section{IN THE NEWS}

Signs of evolution

The discovery that deaf students in Nicaragua have developed a new sign language generated a great deal of interest after it was published in Science. According to CBC News (17 September), "Students attending the country's first school for the deaf in Managua weren't taught sign language, but they invented their own way to communicate, giving scientists insight into how we learn language."

The study was led by Ann Senghas of Columbia University, New York, USA. The opportunity to study the evolution of a new language over a relatively short timescale has allowed the researchers to investigate how much of our linguistic ability is innate.

The researchers found that older users of the new language used a relatively simple form, without formal grammar - whereas younger students used discrete words to form sentences in a way that resembled other languages. "Their first pantomine-like gestures evolved into a grammar of increasing complexity as new children learned the signs and elaborated. Now it has a formal name: Nicaraguan Sign Language, and is so distinct that it would not be understood by American and British signers." (Daily Telegraph, UK, 17 September).

Psychologist Steven Pinker, speaking to $B B C$ News Online, was impressed. "I think this research has made some of the most interesting discoveries in language acquisition in decades. It shows that children have sophisticated mechanisms of language analysis which give language many of its distinctive qualities."

\title{
Astrocytes grab a piece of the action
}

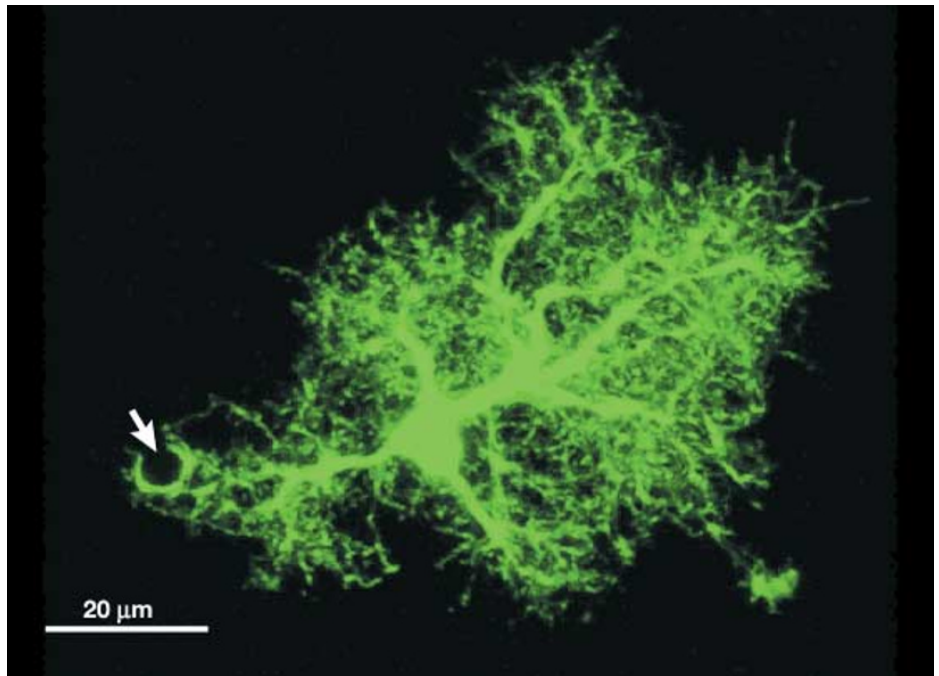

An EGFP-expressing astrocyte in the cortex of a one-week-old mouse. On one side of this highly polarized cell, an astroglial endfoot contacts a brain capillary (arrow), and on the other side, its highly branched processes enwrap the numerous synapses of the brain parenchyma. Image courtesy of F. Kirchhoff, Max Planck Institute of Experimental Medicine, Göttingen, Germany. See online supplementary information S1 (movie).

\section{Reconciling trafficking models}

NMDA ( $N$-methyl-D-aspartate) receptor (NMDAR) clusters are found on the surfaces of dendrites before the formation of glutamatergic synapses, but how do they get there? The popular view is that they are transported along microtubules in carrier vesicles, and are exocytosed to the dendritic surface only when they reach their destination. However, they might also be incorporated into the plasma membrane and travel by lateral diffusion. In the Journal of Neuroscience, Washbourne and colleagues present a new model that incorporates aspects of both of these hypotheses.

The authors cultured cells from the newborn rat visual cortex for 3-4 days to bring them to the stage just before synaptogenesis, and they immunostained these living neurons with an antibody that recognized the extracellular portion of the NMDAR subunit NR1. They found that they could detect NMDAR clusters on the surface of these young neurons, and each cluster contained both surface-exposed and internal receptors. The authors also transfected neurons with a construct that encoded NR1 fused to an extracellular enhanced green fluorescent protein (EGFP). By labelling live neurons with an antiGFP antibody, they showed that about $90 \%$ of EGFP-NR1 clusters were exposed to the dendritic surface at some point during a 20 minute imaging period. This 'surface-labelled' NR1 was mobile and could travel along dendrites, although the trafficking mechanism could not be determined from these experiments.

Do the surface-labelled EGFP-NR1 clusters remain on the
Recent imaging studies have indicated that dendritic spines can be highly motile during synaptic development and plasticity, and according to a new report in the European Journal of Neuroscience, astrocytic processes are probably just as dynamic.

Astrocytes are no longer considered to be a passive glue that connects and supports neurons, and there is growing evidence that they are important modulators of synaptic transmission. Astrocytic processes have been shown to be intimately associated with synapses, so they are also ideally placed to synchronize neuronal network activity.

In the new study, Hirrlinger, Hülsmann and Kirchhoff used timelapse imaging in slices of mouse brainstem to monitor the movements

dendritic surface, or are they endocytosed? To investigate, Washbourne et al. labelled surface proteins with biotin, then stripped the biotin from the cell surface after various periods of time. After a 5minute incubation period, about $50 \%$ of the surface-labelled NR 1 subunits had remained biotinylated, indicating that a substantial proportion had undergone endocytosis during this time.

Next, Washbourne et al. used a pulse-chase approach to explore whether the endocytosed EGFP-NR1 clusters were returned to the neuronal surface. They surface labelled EGFP-NR1transfected neurons with anti-GFP antibody and incubated the cells to allow internalization of the EGFP-NR1 clusters to take place. Next, they removed the surface antibody with acid, and, after a further incubation, they labelled the neurons with a secondary 'antianti-GFP' antibody. The secondary antibody detected only receptor subunits that were inside the cell at the time of the acid wash and that were subsequently exocytosed. 
RESEARCH H IGHLIGHTS

of astrocytic processes in the vicinity of active synapses. To visualize astrocytes and their processes, the authors used a transgenic mouse line, in which enhanced green fluorescent protein (EGFP) was expressed under the control of the promoter for the astrocyte-specific marker glial fibrillary acidic protein (GFAP). They tagged active synapses by staining the tissue with FMI-43, a fluorescent dye that is taken up by recycling neurotransmitter vesicles.

The authors were able to identify astrocytic processes that were in close contact with active synapses. These processes showed two types of movement: they formed lamellipodia-like structures that could glide across the surfaces of neurons, and they extended processes that resembled filopodia.

What might be the functional significance of astrocytic-process motility at synapses? Hirrlinger et al. suggest that it might contribute to the appropriate presentation of important

The authors found that a large proportion of the internalized subunits were returned to the dendritic surface.

Using immunogold labelling and electron microscopy, the authors showed that some NR1 subunits were found in tubulovesicular structures that were associated with microtubules. Therefore, their data indicate a model in which NMDARs undergo intracellular trafficking along microtubules, which is punctuated by cycles of exocytosis and endocytosis. However, the possibility of a parallel lateraldiffusion mechanism cannot be ruled out at this stage.

What might be the purpose of the endocytic-exocytic cycling? regulatory molecules. For example, one of the ways in which astrocytes are thought to regulate neurotransmission is through the release and uptake of glutamate, and the reorientation of astrocytic processes might allow the correct positioning of glutamate transporters and release sites.

Thanks to these new findings, the idea that astrocytes have an active role in synaptic function takes on a more literal meaning.

(D) References and links ORIGINAL RESEARCH PAPER Hirlinger, J. et al. Astroglial processes show spontaneous motility at active synaptic terminals in situ. Eur. J. Neurosci. 20, 2235-2239 (2004)

FURTHeR READING Bezzi, P. et al. Astrocytes contain a vesicular compartment that is competent for regulated exocytosis of glutamate. Nature Neurosci. 7, 613-620 (2004) | Haydon, P. G. Glia: listening and talking to the synapse. Nature Rev. Neurosci. 2, 185-193 (2001)| Newman, E. A. New roles for astrocytes: regulation of synaptic transmission. Trends Neurosci. 26, 536-542 (2003)

WEB SITE

Encyclopedia of Life Sciences:

http://www.els.net/

astrocytes and brain signalling

Washbourne et al. propose that it could allow the sorting of different types of glutamate receptor; for example, NMDARs and AMPA ( $\alpha$-amino-3-hydroxy5-methyl-4-isoxazole propionic acid) receptors might be exocytosed simultaneously but endocytosed differentially. Also, the expression of glutamate receptors at the dendritic surface could influence the formation of glutamatergic synapses by enabling the neuron to detect exogenous glutamate.

Heather Wood

(9) References and links ORIGINAL RESEARCH PAPER Washbourne, $P$. et al. Cycling of NMDA receptors during trafficking in neurons before synapse formation. J. Neurosci. 24, 8253-8264 (2004)

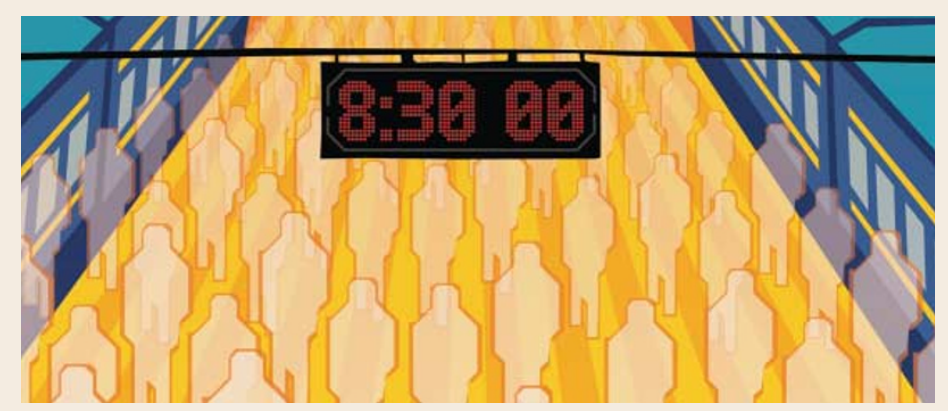

\section{IN BRIEF}

\section{REPAIR}

Nerve regeneration and wound healing are stimulated and directed by an endogenous electrical field in vivo.

Song, B. et al. J. Cell Sci. 117, 4681-4690 (2004)

Endogenous, extracellular physiological electric fields were discovered over a century ago, but little is known about their effects on biological processes. In this study, the authors show that wounding the cornea generated a laterally orientated electric field under the epithelium, and that pharmacological manipulation of the electric field affected nerve sprouting, the direction of nerve growth and the rate of epithelial wound healing.

\section{PAIN}

Electrophysiological properties of mutant $\mathrm{Na}_{\mathrm{v}} 1.7$ sodium channels in a painful inherited neuropathy.

Cummins, T. R. et al. J. Neurosci. 24, 8232-8236 (2004)

Dysregulated expression of sodium channels has been associated with neuropathic pain, but no mutations have been identified that lead to changes in sodium channel function and increased pain sensation. Two independent point mutations in $\mathrm{Na}_{\mathrm{v}} 1.7$ are linked to familial primary erythermalgia - a dominantly inherited painful neuropathy. This study shows that these mutations cause a significant hyperpolarizing shift in activation and slow deactivation. The mutations also result in an increase in amplitude of the current produced by $\mathrm{Na}_{\mathrm{v}} 1.7$ in response to slow, small depolarizations.

\section{NEURODEGENERATIVE DISORDERS}

Nonsteroidal anti-inflammatory drugs lower $A \beta_{42}$ and change presenilin 1 conformation.

Lleó, A. et al. Nature Med. 26 September 2004 (10.1038/nm1112)

Some commonly used nonsteroidal anti-inflammatory drugs (NSAIDs) can shift the cleavage products of amyloid precursor protein (APP) to a shorter, less fibrillogenic form, but the underlying mechanism is not known. Lleó and colleagues show that NSAIDS can change the conformation of presenilin 1 - the $\gamma$-secretase that cleaves APP — which alters APP-presenilin 1 interaction and modifies the cleavage site.

\section{VISUAL SYSTEM}

\section{Small modulation of ongoing cortical dynamics by sensory input during natural vision.}

Fisher, J. et al. Nature 431, 573-578 (2004)

This study examines the relationship between spontaneous cortical activity and the response of primary cortical neurons during natural viewing conditions in ferrets. The correspondence between evoked neural activity and the input signal structure was weak in young animals, but improved with age. At all ages, correlations in spontaneous neural firing were only slightly modified by visual stimulation, irrespective of the sensory input. The results indicate that, during sensory coding, stimulus-evoked activity in the visual cortex might reflect modulation of intrinsic circuits by sensory signals, instead of directly encoding the structure of the input signal. 